SLAC-PUB-7247

February 1999

\title{
The Next Linear Collider Test Accelerator s RF Pulse Compression and Transmission Systems
}

\author{
S. G. Tantawi et al.
}

Presented at the 5th European Particle Accelerator Conference (EPAC 96), Sitges, Spain, June 10-14, 1996

Stanford Linear Accelerator Center, Stanford University, Stanford, CA 94309

Work supported by Department of Energy contract DE AC03 76SF00515. 


\title{
The Next Linear Collider Test Accelerator's RF Pulse Compression and Transmission Systems
}

\author{
S.G.Tantawi, C. Adolphsen, S. Holmes ,T. Lavine, R.J. Loewen, C. Nantista, C. Pearson, R. Pope, \\ J.Rifkin, R.D. Ruth, A.E. Vlieks \\ Stanford Linear Accelerator Center, Stanford University, Stanford, CA, 94309, U.S.A.
}

\section{Abstract}

The overmoded rf transmission and pulsed power compression system for SLAC's Next Linear Collider (NLC) program requires a high degree of transmission efficiency and mode purity to be economically feasible. To this end, a number of new, high power components and systems have been developed at X-band, which transmit rf power in the low loss, circular TE01 mode with negligible mode conversion. In addition, a highly efficient SLED-II* pulse compressor has been developed and successfully tested at high power. The system produced a $200 \mathrm{MW}, 250 \mathrm{~ns}$ wide pulse with a near-perfect flat-top. In this paper we describe the design and test results of the high power pulse compression system using SLED-II.

\section{1††INTRODUCTION}

The NLC rf systems use low loss highly over-moded circular waveguides operating in the $\mathrm{TE}_{01}$ mode. The efficiency of the systems is sensitive to the mode purity of the mode excited inside these guides. We used the so called flower petal mode transducer [2] to excite the $\mathrm{TE}_{01}$ mode. This type of mode transducer is efficient, compact and capable of handling high levels of power.

To make more efficient systems, we modified this device by adding several mode selective chokes to act as mode purifiers. To manipulate the rf signals we used these modified mode converters to convert back and forth between over-moded circular waveguides and single-moded WR90 rectangular waveguides. Then, we used the relatively simple rectangular waveguide components to do the actual manipulation of rf signals. For example, two mode transducers and a mitered rectangular waveguide bend comprise a 90 degree bend. Also, a magic tee and four mode transducers would comprise a four-port-hybrid, etc. We will discuss the efficiency of an rf transport system based on the above methodology.

We also used this methodology in building the SLED-II pulse compression system. At SLAC we built 4 of these pulse systems. In this paper we describe the SLED-II system and compare the performance of these 4 systems at SLAC. We report the experimental procedures used to measure their performance as well as the results of high power tests.

\section{$2 \uparrow+$ CHOKED MODE CONVERTER}

The flower petal mode converter is described in details in [2]. The measured level of power in spurious TE modes is $\sim 0.5 \%$. Simulations show similar levels of contamination due to TM modes. Although these levels of contamination are small, experimental measurements observed in a transmission line composed of a $7.442 \mathrm{~cm}$ diameter waveguide surrounded by two mode converters are high.

These losses are due to the mode conversionreconversion phenomenon observed in over-moded waveguides used in communication systems[3]. Since the mode converter is efficient, for a spurious mode the circular waveguide with the two mode converters represents a high quality factor cavity. Because the line is large compared to the wavelength, there is a large number of resonating modes in the frequency band of interest. These resonant modes are coupled to the input signal because of the small mode contamination produced by the mode converters.

We basically have two choices in order to reduce the mode conversion losses. We can either reduce the coupling to spurious modes by improving the mode converter or reduce the quality factors of these resonating modes by inserting mode filters. Vacuum compatible mode filters are expensive and hard to make. We therefore tried to improve the performance of the mode converter by using mode selective chokes.

According to [2] the modes $\mathrm{TE}_{11}, \mathrm{TE}_{41}$, and $\mathrm{TM}_{11}$ represent the main components of the mode contamination produced by the flower petal. The output waveguide of the mode converter has a diameter of $4.737 \mathrm{~cm}$. Except for the $\mathrm{TE}_{41}$ mode this guide size will not allow $\mathrm{TE}_{4 \mathrm{n}}$ modes to propagate. Hence, a single choke designed to reflect $\mathrm{TE}_{41}$ mode at the operating frequency of $11.424 \mathrm{GHz}$ will greatly reduce the contamination from this mode. Also, if the choke width along the waveguide axis is less than the free space half wavelength it will not affect the $\mathrm{TE}_{01}$ mode. Since the modes $\mathrm{TE}_{11}, \mathrm{TM}_{11}$, and $\mathrm{TE}_{12}$ have the same azimuthal variations, any choke, designed to reflect any of them, will produce some mode conversion to the other modes. However, several chokes, properly spaced, can act as an effective reflector for all modes.

We added three chokes to the flower petal mode converter, one for the $\mathrm{TE}_{41}$ and two for the $\mathrm{TM}_{11}$ and $\mathrm{TE}_{11}$ modes. This reduced the number and depth of these resonant modes.

\section{$3 \uparrow \uparrow I M P L E M E N T A T I O N$ OF SLED II}

Figure 1 shows the pulse compression system. It uses two 35.35 to 37.5 -meter long cylindrical copper waveguides as delay lines, each $12.065 \mathrm{~cm}$ in diameter and operating in the $\mathrm{TE}_{01}$ mode. In theory, these over-moded delay lines can form 
a storage cavity with a quality factor $\mathrm{Q}>1 \times 10^{6}$. Each of the delay lines is terminated by a shorting plate whose axial position is controllable to within $\pm 12 \mu \mathrm{m}$ by a DC motor with a position-monitor feed back system. The input of the line is tapered down to a $4.737 \mathrm{~cm}$ diameter waveguide at which the mode $\mathrm{TE}_{02}$ is cut-off; hence, the circular irises that determine the coupling to the lines do not excite higher order modes provided that they are perfectly concentric with the waveguide axis. A choked mode converter excites the $\mathrm{TE}_{01}$

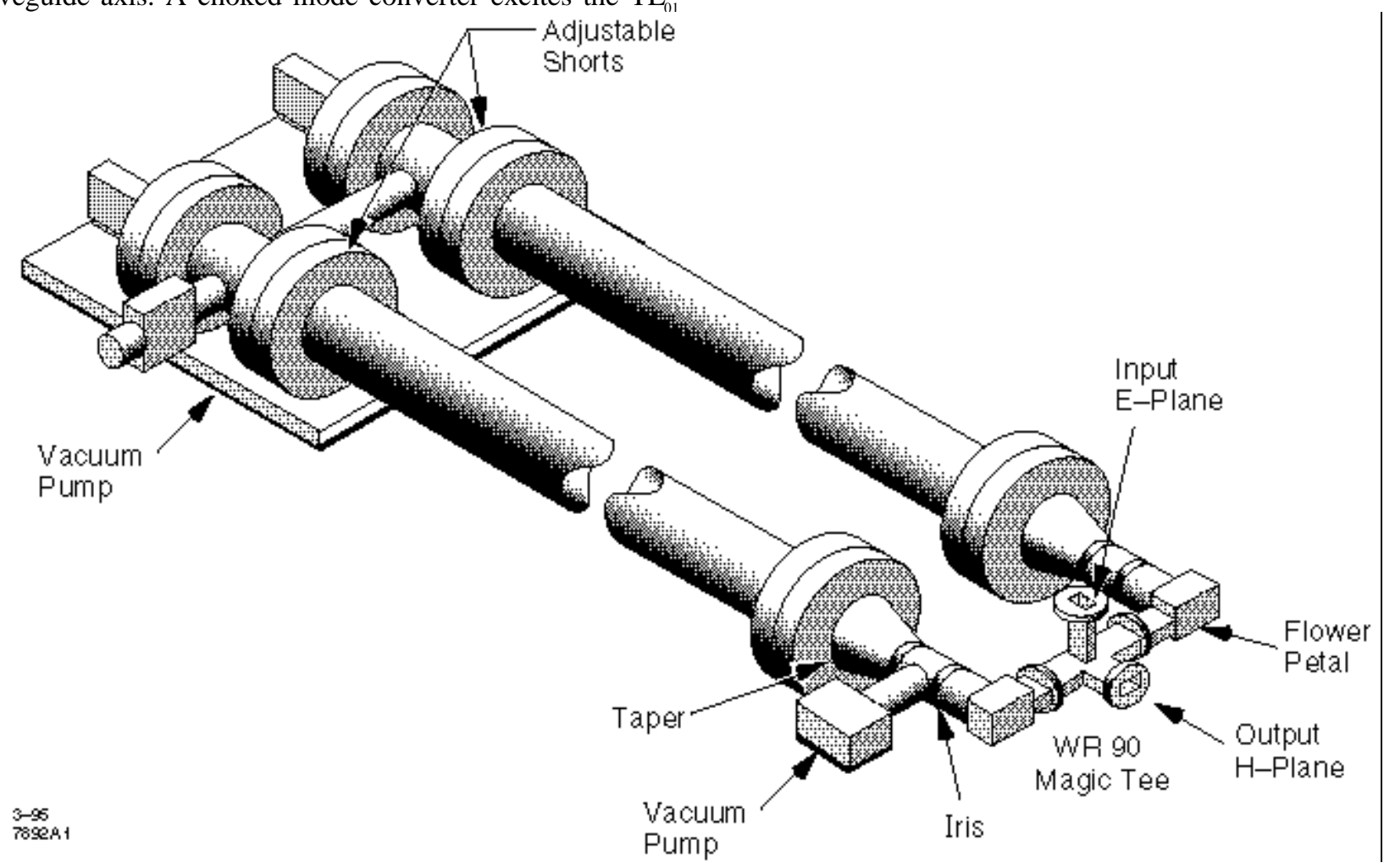

Figure 1. SLED-II Layout. It has two $12.065 \mathrm{~cm}$ diameter waveguides, $35.35 \mathrm{~m}$ in length ( $223 \mathrm{nS}$ round-trip time.)

\section{4††MEASUREMENTS}

All measurements were performed using an HP8510C network analyzer with the results examined in the time domain using a PC. The frequency domain measurements were transferred to the PC via a GPIB link and multiplied by the FFT of a maximally flat pulse modulating an 11.424 $\mathrm{GHz}$ signal. This pulse has a limited frequency response that is smaller than the measurement's frequency span.

The time domain output is produced by taking the IFFT of this frequency domain product. Note that once we obtain the frequency characteristics of the system from the network analyzer, we can calculate the time domain response for any arbitrary input pulse. Furthermore, the phase reversed pulse required for SLED II can be sensitized by linear addition of two maximally flat pulses.

\section{5††EXPERIMENTAL RESULTS}

\subsection{Transmission Line Measurements}

The transmission line that connects the output of the first pulse compressor in the NLCTA to the injector section of mode just before each iris. Both mode converters are connected to the coplanar arms of a high-power WR90 magic tee. The arms differ in length by a quarter wavelength at the operating frequency of $11.424 \mathrm{GHz}$. Therefore, the reflection from the lines exits through the $\mathrm{H}$-arm when the input to the lines enters from the E-arm. The distance from the irises to the center of the magic tee has been adjusted to within $\pm 13 \mu \mathrm{m}$ to maximize this transmission. 


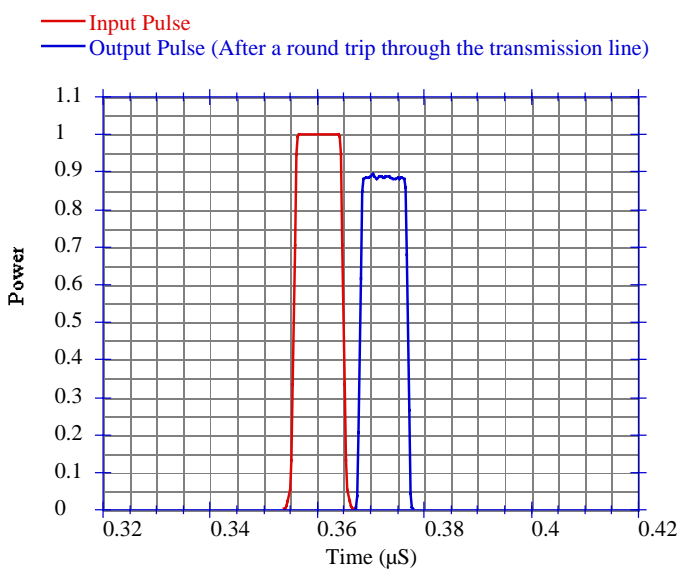

Figure 2. Performance of the transmission line that connects the pulse compressor to the accelerator.

\subsection{SLED II Measurements}

Figure 3 shows the response of the NLCTA injector system to a $1.338 \mu \mathrm{S}$ pulse. The last $223 \mathrm{nS}$ of the pulse have 180 degree phase shift. (A compression ratio of 6). The measured efficiency of the system is $67.5 \%$. It also compares the high power measurements to the cold test results.

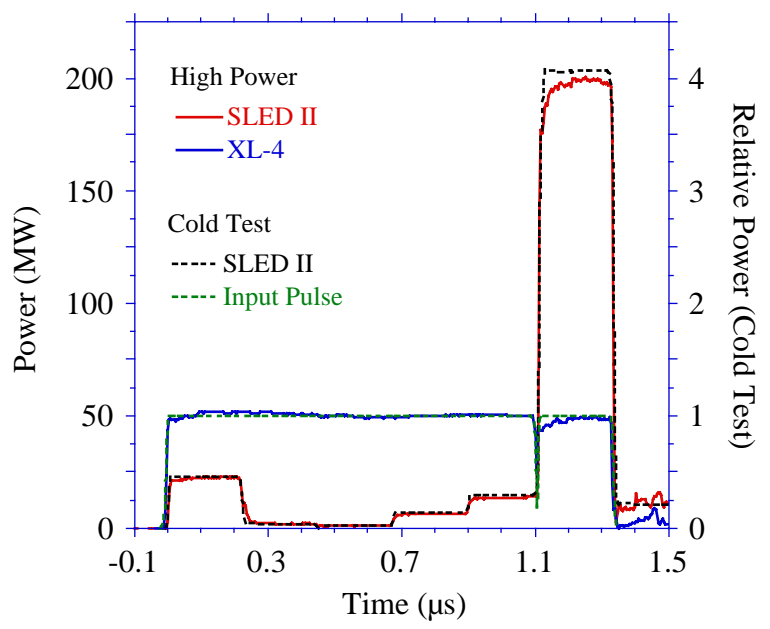

Figure 3. Measured Output of the NLCTAís First RF Pulse Compressor.

The power gain was measured for a series of compression factors. A least-squares fitting of these measurements to theoretical response [4] is shown in Figure 4. The round trip power loss was found to be $1.51 \%$, indicating an intrinsic $Q$ for the lines of $1.05 \times 10^{6}$. The theoretical value for the round trip losses is $1.15 \%$. This low level of losses in the lines indicates that an extremely pure $\mathrm{TE}_{01}$ mode is being excited in these lines. The external losses are $6.89 \%$, and the iris reflection coefficient is 0.70 . The iris was designed using a mode matching code to have a reflection coefficient of 0.685 , the optimum value for a compression ratio of 6 .

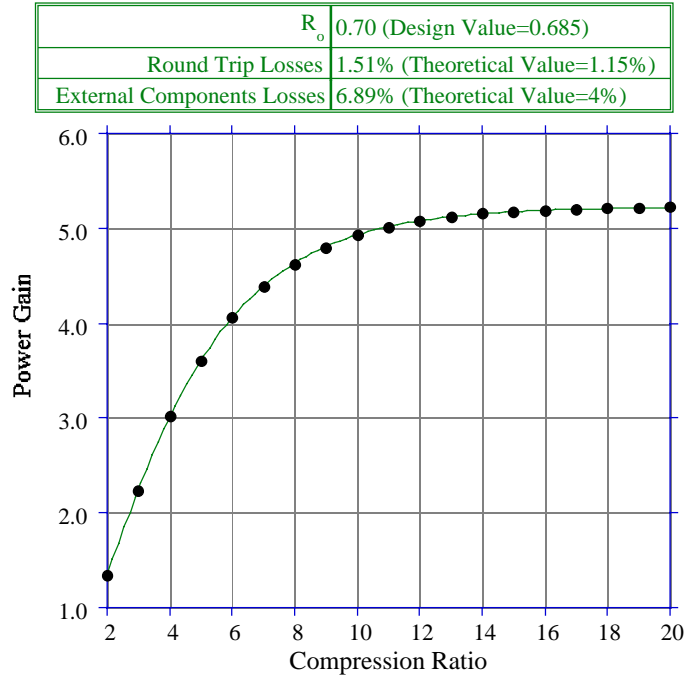

Figure 4 The points are measured power gains. The above table shows the fitting parameters.

This procedure was repeated for all 4 systems built at SLAC. Table I shows a comparison between all these systems.

\begin{tabular}{|c|c|c|c|c|}
\hline & $\begin{array}{c}\text { Test Lab } \\
\text { SLED II }\end{array}$ & $\begin{array}{c}\text { NLCTA } \\
\text { Station } \\
\# 0\end{array}$ & $\begin{array}{c}\text { NLCTA } \\
\text { Station } \\
\# 1\end{array}$ & $\begin{array}{c}\text { NLCTA } \\
\text { Station\# } \\
2\end{array}$ \\
\hline $\begin{array}{c}\text { Compressio } \\
\text { n Ratio }\end{array}$ & 8 & 6 & 6 & 6 \\
\hline $\begin{array}{c}\text { Pulse width } \\
\text { (nS) }\end{array}$ & 150 & 223 & 245 & 245 \\
\hline $\begin{array}{c}\text { Delay Line } \\
\text { Losses/100n } \\
\text { S (\%) }\end{array}$ & 1.63 & 0.68 & 0.52 & 0.59 \\
\hline Intrinsic Q & $4.3 \times 10^{5}$ & $1.05 \times 10^{6}$ & $1.37 \times 10^{6}$ & $1.21 \times 10^{6}$ \\
\hline $\begin{array}{c}\text { Total Delay } \\
\text { Line Losses } \\
(\%)\end{array}$ & 5.05 & 3.63 & 2.34 & 2.39 \\
\hline $\begin{array}{c}\text { External } \\
\text { Losses (\%) }\end{array}$ & 4.85 & 6.89 & 6.36 & 5.26 \\
\hline $\begin{array}{c}\text { Total } \\
\text { Efficiency } \\
(\%)\end{array}$ & $\underline{58.6}$ & 67.5 & 68.6 & 69.3 \\
\hline
\end{tabular}

Table I. Comparison Between the 4 SLED II Systems at SLAC

\section{$6+$ CONCLUSION}

We have demonstrated efficient transport and pulse compression rf systems suitable for the NLC program. These systems are based on an efficient and compact mode transducer. The implemented systems approached the theoretical design values. We also demonstrated measurement techniques capable of measuring rf systems with very small losses. 


\section{$7 \dagger \uparrow A C K N O W L E D G M E N T$}

This work is supported by the US Department of Energy under contract DE-AC03-76F00515.

\section{REFERENCES}

[1] P. B. Wilson, Z. D. Farkas, and R. D. Ruth, "SLED II: A New Method of RF Pulse Compression," Linear Accelerator Conference, Albuquerque, NM, September 1990; SLAC-PUB-5330.

[2] S. G. Tantawi et al., "Numerical Design and Analysis of a Compact TE10 to TE01 Mode Transducer," Conference on Computational Accelerator Physics, Los Alamos, NM, 1993, AIP Conference Proceedings 297, pp. 99-106.

[3] S. E. Miller, ìWaveguide as a Communication Medium,î The Bell System Technical Journal, Nov. 1954, pp. 1209-1265.

[4] S. G. Tantawi et al, ì Active Radio Frequency Pulse Compression Using Switched Resonant Delay Lines,î Nuclear Instruments \& Methods in Physics Research Section A, Vol. 370 (1996) pp. 297-302. 